\title{
Machine Learning Assisted Propeller Design
}

\author{
Harsh Vardhan, Peter Volgyesi, Janos Sztipanovits \\ Institute for Software Integrated Systems, Vanderbilt University, Nashville, TN, USA
}

\begin{abstract}
Propellers are one of the most widely used propulsive devices for generating thrust from rotational engine motion both in marine vehicles and subsonic air-crafts. Due to their simplicity, robustness and high efficiency, propellers remained the mainstream design choice over the last hundred years. On the other hand, finding the optimal application-specific geometry is still challenging. This work in progress report describes application of modern and rapidly developing Machine Learning (ML) techniques to gain novel designs. We rely on a rich set of preexisting parametric design patterns and accumulated engineering knowledge supplemented by high-fidelity simulation models to formulate the design process as a supervised learning problem.

The aim of our work is to develop and evaluate machine learning models for the parametric design of propellers based on applicationspecific constraints. While the application of ML techniques in optimal propeller design is at a very nascent level, we believe that our early results are promising with a potentially significant impact on the overall design process. The ML-assisted design flow allows for a more automated design space exploration process with less dependency on human intuition and engineering guidance.
\end{abstract}

\section{CCS CONCEPTS}

- Computing methodologies $\rightarrow$ Machine learning; • Applied computing $\rightarrow$ Physical sciences and engineering.

\section{KEYWORDS}

Propeller, Machine learning, Random forest regression, Designspace Exploration, OpenProp, Evolutionary Algorithm

\section{PROBLEM FORMULATION AND APPROACH}

A propeller is a propulsion device that converts rotational motion into thrust by forcing the surrounding fluid flow axially, by means of rotating blades mounted in a shaft that is driven by an actuator or engine. Performance of a propeller depends upon its geometrical characteristics and physical parameters. For example, [3] considers the number of blades $(Z)$, propeller's diameter $(D)$, chord radial distribution $(C / D)$, pitch radial distribution $(P / R)$ and hub diameter $(D h u b)$ as geometrical characteristics and thrust coefficient

Permission to make digital or hard copies of all or part of this work for personal or classroom use is granted without fee provided that copies are not made or distributed for profit or commercial advantage and that copies bear this notice and the full citation on the first page. Copyrights for components of this work owned by others than the author(s) must be honored. Abstracting with credit is permitted. To copy otherwise, or republish, to post on servers or to redistribute to lists, requires prior specific permission and/or a fee. Request permissions from permissions@acm.org.

ICCPS '21, May 19-21, 2021, Nashville, TN, USA

(c) 2021 Copyright held by the owner/author(s). Publication rights licensed to ACM ACM ISBN 978-1-4503-8353-0/21/05 . .\$15.00

https://doi.org/10.1145/3450267.3452001
Table 1. Requirement Space and Geometric Design Space

\begin{tabular}{c|c|l|c|c} 
Variable & Minimum & Maximum & Unit & Count \\
\hline Thrust & 100 & 500000 & Newton & 499900 \\
\hline Vel ship & 0 & 20 & $\mathrm{~m} / \mathrm{s}$ & 200 \\
\hline RPM & 50 & 6000 & $\mathrm{rpm}$ & 5950 \\
\hline Diameter & 0 & 10 & $\mathrm{~m}$ & 100 \\
\hline Chord Profile(9) & 0 & 0.5 & - & 500
\end{tabular}

$\left(C_{t}\right)$, power coefficient $\left(C_{p}\right)$ and advance ratio $(J)$ as governing physical parameters. Given some thrust requirements at well-defined operational points (e.g. cruising speed and the actuator's spinning rate $(R P M))$, the goal of a designer is to search for the optimal geometric characteristics than can fulfill these requirements at maximum efficiency $(E)$. In general, this requirement-driven design process is a multi-parameter search and optimization problem in a geometric design space(refer Figure 1). While novel propeller geometries may

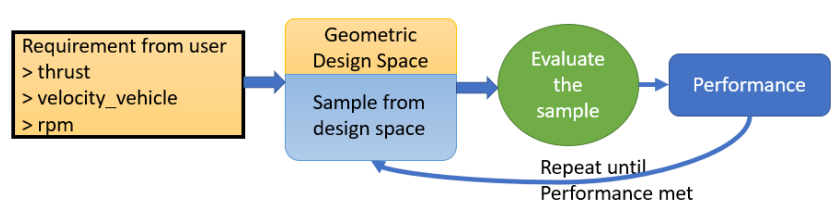

Figure 1: Propeller Design Problem

have significant gains, the design space increases exponentially with the number of parameters describing the geometric characteristics of design points. Search algorithms like constraint satisfaction (CSP) and the propositional satisfiability problem (SAT) cannot be deployed in these kind of settings as feasibility of the design point cannot be established prior to evaluating it. These kind of problems are referred as unknown feasibility problems [4]. In our case, we can split the search space to two parts: (1) requirement space (thrust, velocity of vehicle, rpm), as we want our ML guided design tool to work not only on one specific requirement but for a range of requirements and (2) geometric design space (chord profile radial distribution $(C / D)$, diameter $(D)$, hub diameter $(D h u b)$, etc). Table 1 shows our currently targeted set of requirements and geometric design parameters.

The performance criteria for a design is the efficiency of propeller, if the design converges after optimisation. The goal is to maximise efficiency as much as possible. We define a design to be valid if it converges and its performance metric efficiency $(E)$ is greater than $50 \%$. This threshold of $50 \%$ is derived from domain knowledge as the lowest acceptable efficiency of propellers in large cargo ships is around $50 \%$ [1], while on the other hand propellers used in subsonic airplanes can reach efficiency up to $80-90 \%$ [5].

The requirement space after coarse discretisation is in the order of $10^{11}$ (thrust $\mathrm{x}$ velShip x RPM), while the geometric design 
space explodes even faster and is in the order of $10^{27}$ (diameter * nine different cord radial profile). The size of the combined search space is approximately $10^{38}$. A significant problem with the manual exploration process is that previous results are forgotten and not used to their fullest power in the consecutive search steps. Our approach is to accumulate as much information and knowledge as possible by systematic search in the design space. After collecting all valid design points by storing the requirement values $(R)$ with the corresponding geometric design values $(G)$ and resulting efficiency $(E)$, the goal is to learn a function $f: R \mapsto G, E$. The primary motivation for learning a function approximation is to manage the inherently huge search space, where naïve search is not feasible. By learning a manifold function $f$, we can interpolate and generalise between sampled design point. This function $f$, is called PropDesigner, and divides the design process in two phases (refer figure 2). On a given requirement, in Phase I, the learned predictor $f$ captured by PropDesigner predicts the geometric design and associated efficiency. We use this predicted design and associated efficiency as baseline and guide the process to phase II, where we search in geometric design space to further improves the design by running a trace of evolutionary algorithm process to improve the design in the local geometric region. For this purpose we take a very small step (hyperparamter) in each dimension of the geometric region and evaluate all the possible design points locally, and select the best one and reject all others. We repeat this process for a small number of episodes, only if efficiency is improving in any dimension. It is done in hope to find local best design by spending very little of computation(refer fig 2).

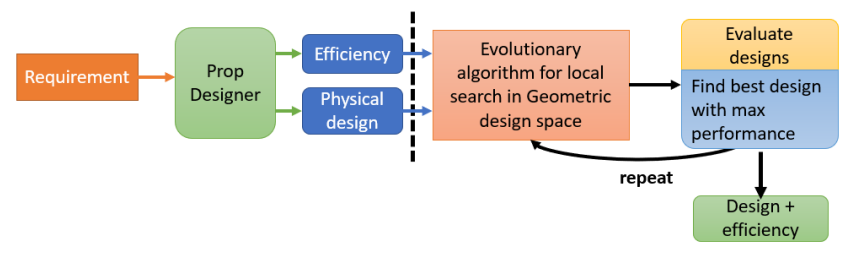

Figure 2: Assistive Design

\section{DATA GENERATION AND TRAINING}

For data generation, we are using an open-source parametric design and analysis tool called OpenProp [3], which evaluates different design and requirement points in the search space. We sample one point from both requirement space and physical design space and give these as an input to the optimiser of OpenProp. OpenProp internally optimises this design point and computes the performance metric. We store these valid design points as our design corpora. Till now, we have sampled 10 million design points and found 0.205 million valid design points. Using this design corpus, we trained a random forest regression[2] model on our dataset, with the requirements as inputs and the geometric design parameters and efficiency metric as outputs. With the collected 205556 data points, rather than splitting the data in training and test set, we took the route of 10 -fold cross-validation. The selected random forest model is an ensemble of 100 decision trees with mean squared error (MSE) as splitting criteria of the node. In our future work, we will build and evaluate more complex neural network-based approximators for this prediction task.

\section{PRELIMINARY RESULT DEMONSTRATION}

At the preliminary stage of data collection, model development, training, and initial evaluation, there are two interesting results to share: (1) the discovery of unconventional propeller geometric design with very high efficiency and (2) the prediction accuracy of the PropDesigner. Our first result is that we discovered 9851 designs have an efficiency above $80 \%$ which is very high for marine propellers[1]. One of the design with an efficiency of $90 \%$ on a given requirement is shown in figure 3 . Another interesting fact about this predicted design is its unconventional geometric design from the currently used propeller with very high efficiency.
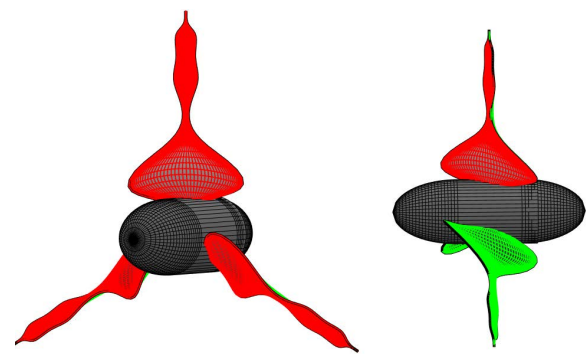

Figure 3: Propeller with efficiency 90\% (producing 30600 Newton of thrust at cruising speed of $14 \mathrm{~m} / \mathrm{s}$ with rotation rate $338 \mathrm{rpm}$ )

Our second result is that after training the random forest regression model and testing it on the rest $10 \%$ validation data (20556 requirements), we found that in expectation on $80.2 \%$ of requirement inputs, the predicted design has performance metric 'efficiency' is within $7.5 \%$ of error value from prediction done by OpenProp and by predictor PropDesigner. This accuracy on validation data is exciting and these results give a hope that further progress in this direction may help to explore new unseen efficient designs and save huge time for system designers along with challenges to validate these designs beyond the used tool by high fidelity analysis.

\section{ACKNOWLEDGMENTS}

This work is supported by DARPA's Symbiotic Design for CPS project and by the Air Force Research Laboratory (FA8750-20-C0537). Any opinions, findings, and conclusions, or recommendations expressed in this material are those of the author(s) and do not necessarily reflect the views of DARPA or AFRL.

\section{REFERENCES}

[1] 2019. ShipPropulsion. https://spain.mandieselturbo.com/docs/librariesprovider10/ sistemas-propulsivos-marinos/basic-principles- of-ship-propulsion.pdf

[2] Leo Breiman. 2001. Random forests. Machine learning 45, 1 (2001), 5-32.

[3] Brenden Epps, Julie Chalfant, Richard Kimball, Alexandra Techet, Kevin Flood, and Chrysssostomos Chryssostomidis. 2009. OpenProp: An open-source parametric design and analysis tool for propellers. In Proceedings of the 2009 grand challenges in modeling \& simulation conference. 104-111.

[4] Michael A Gelbart, Jasper Snoek, and Ryan P Adams. 2014. Bayesian optimization with unknown constraints. arXiv preprint arXiv:1403.5607 (2014).

[5] Frank Hitchens. 2015. Propeller aerodynamics: the history, aerodynamics \& operation of aircraft propellers. Andrews UK Limited. 